Publ. Astron. Soc. Aust., 1997, 14, 73-6

\title{
The MOST Galactic Plane Survey
}

\author{
A. J. Green \\ School of Physics, University of Sydney, \\ NSW 2006, Australia \\ agreen@physics.usyd.edu.au \\ Received 1996 October 28, accepted 1996 November 15
}

\begin{abstract}
The Molonglo Observatory Synthesis Telescope (MOST) has been upgraded to increase the diameter of the field of view from $70^{\prime}$ to $2 \cdot 7^{\circ}$. A survey of the Galactic Plane is proposed to completely cover the region $240^{\circ} \leq l \leq 365^{\circ},|b| \leq 10^{\circ}$ with a $(1 \sigma)$ sensitivity of $1-2 \mathrm{mJy}$. The results will provide a major resource for the investigation of supernova remnants, $\mathrm{H}$ II regions and transient radio sources. They will also be correlated with the galaxy search from the Hi multibeam survey to be conducted at the Parkes telescope.
\end{abstract}

Keywords: radio continuum survey — Galactic emission — galaxies

\section{Introduction}

A survey of the Galactic Plane is planned with the Molonglo Observatory Synthesis Telescope (MOST), operated by the Astrophysics Department of the University of Sydney. The telescope has recently been converted from operation with a field of view of diameter $70^{\prime}$, to a new wide-field mode with a field size of diameter $2.7^{\circ}$, while retaining its current resolution $\left(\sim 43^{\prime \prime}\right)$ and sensitivity $(1-2 \mathrm{mJy})$. This increase enables a large area of the sky to be surveyed in a relatively short period. Only radio telescopes can observe the region very close to the Galactic Equator since obscuration from dust and high stellar density effectively make the area opaque or heavily confused to optical and infrared telescopes. This survey will complement the northern radio surveys currently in progress. It is anticipated that the data will be made available to the wider astronomical community while the survey is being completed.

\section{Characteristics of the MOST Survey}

The area of the Galactic Plane to be surveyed is $240^{\circ} \leq l \leq 365^{\circ},|b| \leq 10^{\circ}$. Operating in right-hand circular polarisation at $843 \mathrm{MHz}$, the MOST has a resolution of $43^{\prime \prime} \times 43^{\prime \prime} \operatorname{cosec} \delta$, a positional accuracy of about $1^{\prime \prime}$ and now a field size of $2.7^{\circ} \times 2.7^{\circ}$ $\operatorname{cosec} \delta$. The effective thermal noise level $(1 \sigma)$ after a 12 hour observation is $1-2 \mathrm{mJy}$ for the Galactic Plane. For more than $90 \%$ of the surveyed region it is expected that sources of flux density $\geq 5 \mathrm{mJy}$ will be reliably measured. Near strong sources the sensitivity will be constrained by the dynamic range of the telescope, which is about $250: 1$.

\section{Technical Aspects of the Wide-field Upgrade}

The MOST, unlike other earth-rotation synthesis telescopes, forms a bank of hard-wired fan beam responses in real time, without recording the complex visibilities for later processing. The current upgrade has increased by more than a factor of 5 the area of sky which can be fully synthesised in a 12 hour observation. This has been achieved by implementing several innovative improvements, to avoid degrading the sensitivity, while retaining many of the preexisting telescope systems (Robertson 1991; Large et al. 1994). The resolution has been unaffected by these changes. The two principal strategies taken to maintain the sensitivity are the installation of 352 high-performance, low-noise $(12 \mathrm{~K}$ ) ambient temperature preamplifiers, and the development of a system to continuously adjust the phase gradient across the array during observation. The wide-field mode of operation involves a progressive offsetting of the primary beam to several different pointing positions. This beam multiplexing requires a complex phasing system organised by a network of microcontrollers, linked to a central computer. It is expected that commissioning tests will be completed by October 1996. After modification of the data analysis system, the proposed survey will commence.

\section{Scientific Goals}

Radio-frequency observations of the Galactic Plane are particularly valuable because they are unaffected by obscuring dust and high stellar densities. The MOST can image in detail large-scale extended structure and, operating at $843 \mathrm{MHz}$, it is well placed to detect nonthemal emission. A $3^{\circ}$-wide strip centred on the Galactic Equator ( $15 \%$ of the proposed new survey) has been observed with the MOST over the past decade (Green et al. 1997, in preparation). This strip will be repeated providing a crucial second-epoch database for use in studies of long-term radio variability. Figure 1 shows a $3^{\circ} \times 3^{\circ}$ mosaic from the first-epoch Galactic Plane survey 


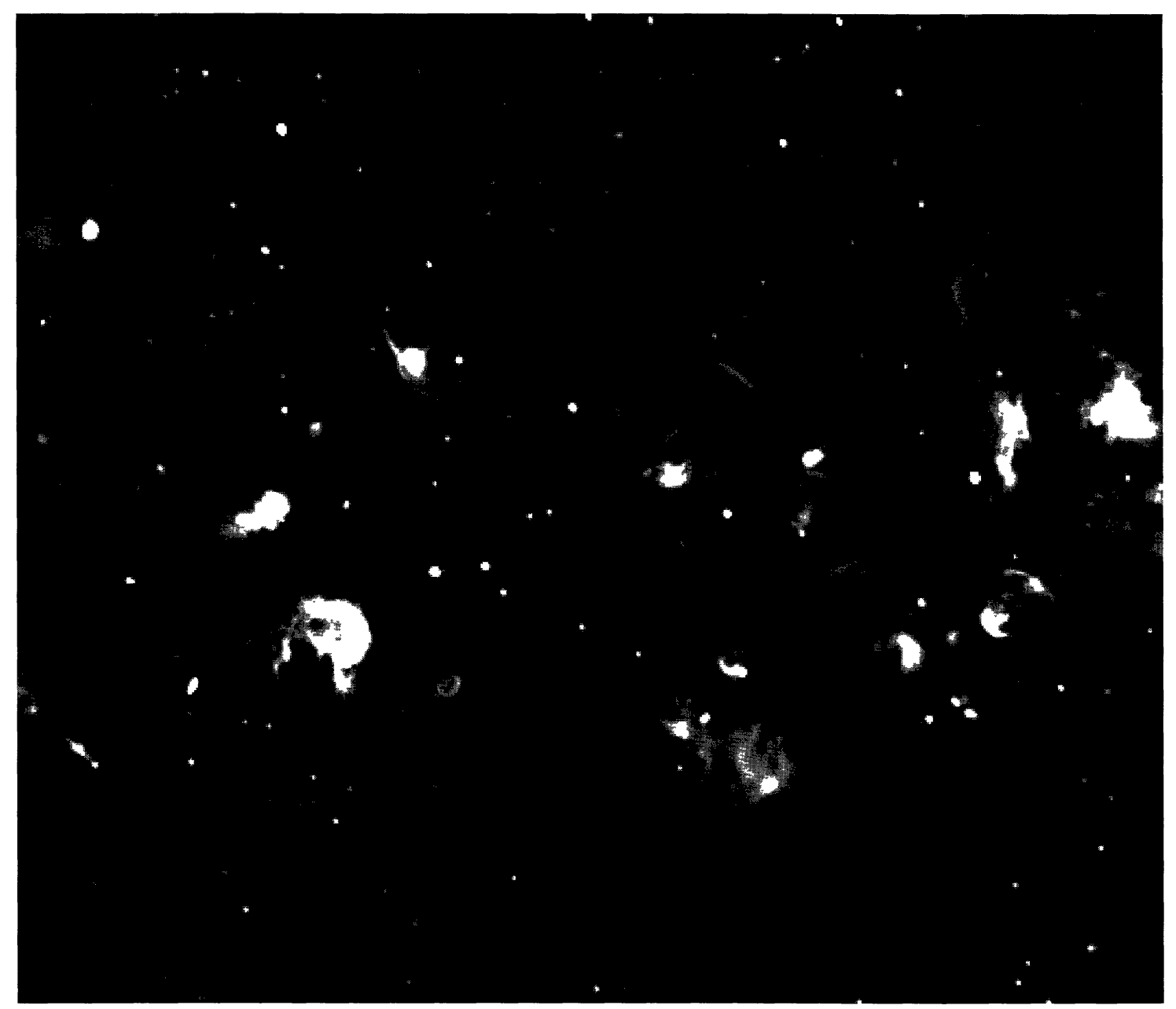

Figure 1-Mosaic of $3^{\circ} \times 3^{\circ}$ region of MOST Galactic Plane survey, centred on $l=318 \cdot 5^{\circ}, b=0 \cdot 0^{\circ}$. Dominant structure is from several Galactic supernova remnants and $\mathrm{H}_{\mathrm{II}}$ regions. Minimum reliable source flux density is $5 \mathrm{mJy}$. The brightest filaments are typically $\sim 80 \mathrm{mJy}$. To show the weak extended emission clearly the greyscale intensity range is saturated above $40 \mathrm{mJy}$.

to illustrate the variation of intensities and angular scale typically seen in MOST observations. Some of the scientific goals of the new survey include:

- New identification of supernova remnants (SNRs). In the first-epoch survey, there were 20 previously unidentified remnants. Given the typical scale-heights of SNRs, it is estimated that an additional $20-30$ candidates will be found.

- Investigation of the large-scale Galactic structure, particularly the filamentary emission seen near complex $\mathrm{H}$ II regions.

- A systematic study of the population of small-diameter Galactic sources as viewed by the MOST. This sample may include planetary nebulae, compact $\mathrm{H}_{\mathrm{II}}$ regions, pulsars and young SNRs. Preliminary work has been done by Whiteoak (1993).
- A program to monitor various types of transient sources. Although variability at $843 \mathrm{MHz}$ is not a common phenomenon, these sources are often of great astrophysical significance. At present long-term radio variability is not well understood. Timescales will be monitored from years down to a few minutes.

Crucial to achieving these goals is the ability to determine the nature of the objects detected by the survey. Spectral index estimates can be made by comparing the MOST data with results from the $1.4 \mathrm{GHz}$ NVSS survey currently in progress at the Very Large Array (Condon et al. 1993). This will be possible for observations north of $\delta=-40^{\circ}$. For the remaining $70 \%$ of the survey there are no large-scale radio frequency databases available with the subarcmin resolution necessary to avoid confusion effects 


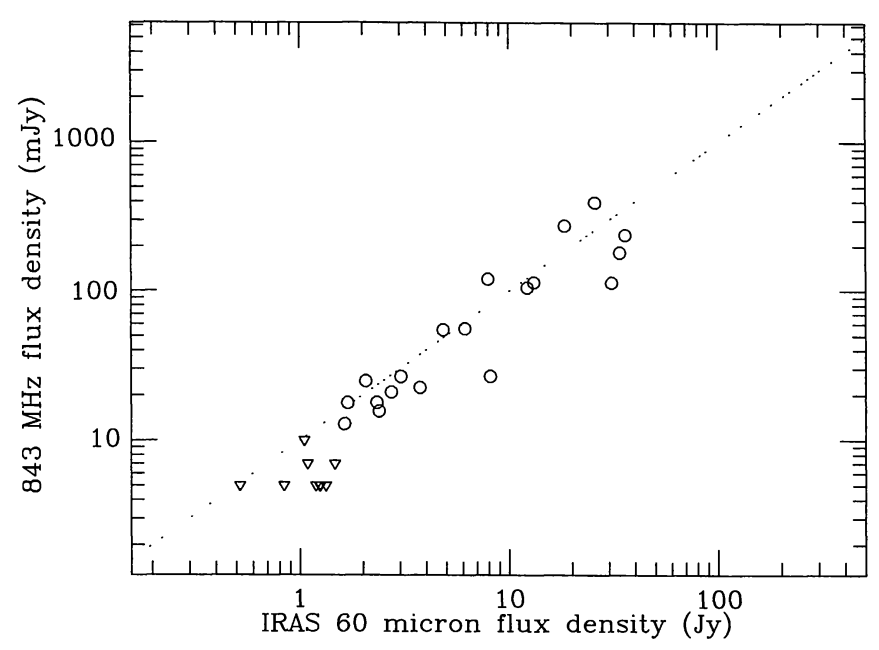

Figure 2-Plot showing IRAS $60 \mu \mathrm{m} /$ radio $843 \mathrm{MHz}$ correlation for spiral galaxies. The line of best fit represents a ratio estimated at 100. Triangles are upper limits at $843 \mathrm{MHz}$.

close to the Galactic Plane. However, it has been found that the principal emission mechanism for Galactic objects can be determined as thermal or nonthermal by comparing their significantly different strengths of $60 \mu \mathrm{m}$ intensity relative to their $843 \mathrm{MHz}$ flux densities (Whiteoak \& Green 1996). Thermal sources are relatively much stronger sources of infrared emission. Structural morphology for extended sources can also give a strong indication of the nature of the object.

For the small-diameter sources, the expected Galactic source density can be calculated by subtracting the contribution from the nearly isotropic distribution of background galaxies. From deep surveys away from the Galactic Plane (Subrahmanya \& Mills 1987), the density of extragalactic objects is expected to be 37 sources/square degree ( $\geq 5 \mathrm{mJy}$ ). Individual source identifications will require additional data at infrared, radio and (where possible) optical frequencies.

As well as investigating the Galactic sources, this survey will also contain much valuable data on the population of extragalactic objects located behind the Plane. Because of the obscuring Galaxy, identification of these sources may be difficult. For extended sources (angular diameter $\geq 4^{\prime}$ ) classical radio galaxy morphologies such as double-lobe, headtail and wide-angle-tail will be recognisable. Large spiral galaxies will be less easy to identify as their structures are likely to be centrally peaked with diameters of only a few minutes of arc. To determine which of the continuum sources are most probably spiral galaxies, the tight correlation between the far-infrared and radio emission from these objects can be used. The relation for $60 \mu \mathrm{m}$ and $843 \mathrm{MHz}$ data is shown here in Figure 2 (Sadler 1996). The line of best fit gives a value of 100 for the ratio between the emission at the two frequencies.

To test the viability of the above methods in identifying extragalactic objects, a pilot study of a small area $\left(3^{\circ} \times 3^{\circ}\right)$ of the first-epoch survey was made (Juraszek 1995). For this region, centred on $l=276 \cdot 5^{\circ}, b=0 \cdot 0^{\circ}$, the B-band extinction is about 4.5 magnitudes, which limits the optical identification sensitivity. An investigation of source densities and the effectiveness of this galaxy identification process is continuing. Preliminary results are promising.

\section{Connection with $H_{\text {I }}$ Multibeam Survey}

A major project to conduct an $\mathrm{H}$ I search for galaxies, out to a distance of $14000 \mathrm{~km} \mathrm{~s}^{-1}$, is in progress using the new multibeam receiver package at the Parkes telescope (Staveley-Smith 1997, present issue p. 111). A strip along the Galactic Plane will receive particular attention. Correlation of the MOST $843 \mathrm{MHz}$ continuum data with the $\mathrm{H}_{\mathrm{I}}$ detections from the multibeam survey will give new information on the radio properties of gas-rich galaxies. It is expected that there will be $\sim 2.6$ MOST sources ( $\geq 5 \mathrm{mJy}$ ) with positional accuracy of $\sim 1^{\prime \prime}$, per Parkes beam, taken as $15^{\prime}$. Estimations show that there will be a detectable MOST source in the radio continuum for the vast majority of the new $\mathrm{H}$ I detections. To identify which of the continuum sources correspond to galaxy candidates, the results will be correlated with the IRAS database. Some additional information may be gained from the shape of the $\mathrm{H}$ I profile, which varies in a systematic way depending on the radial displacement of the galaxy from the Parkes pointing centre (Henning 1997, present issue p. 21). The particular profile shape will enable us to predict which MOST source is most likely the counterpart. 


\section{Conclusions}

The planned Galactic Plane survey with the MOST will yield $>80000$ sources with flux density at $843 \mathrm{MHz}$ of $\geq 5 \mathrm{mJy}$. It will cover the region $240^{\circ} \leq l \leq 365^{\circ},|b| \leq 10^{\circ}$. There will be many new identifications of Galactic SNRs and $H$ II regions and important information on the radio variability of sources. As well, galaxies located in this region of sky will be studied. Strategies are being developed to recognise these objects, where optical identification is restricted. The results will be correlated with the H I multibeam survey at Parkes, and will provide positions accurate to $\sim 1^{\prime \prime}$ for followup work.

\section{Acknowledgments}

A project of this magnitude would not be possible without considerable additional effort from the technical staff at the telescope (under Duncan Campbell-Wilson) and active assistance from all members of the Astrophysics Department. The MOST is operated with the support of the Australian Research Council and the Science Foundation for Physics.

Condon, J. J., Cotton, W. D., Greisen, E. W., Perley, R. A., Yin, Q. F., \& Broderick, J. J. 1993, Bull. AAS, 183, @ 64.02

Henning, P. A. 1997, PASA, 14, 21

Juraszek, S. 1995, Physics Honours Report, University of Sydney

Large, M. I., Campbell-Wilson, D., Cram, L. E., Davison, R. G., \& Robertson, J. G. 1994, PASA, 11, 44

Robertson, J. G. 1991, Aust. J. Phys., 44, 729

Sadler, E. M. 1996, private communication

Staveley-Smith, L. 1997, PASA, 14, 111

Subrahmanya, C. R., \& Mills, B. Y. 1987, IAU Symposium 124, Observatonal Cosmology, ed. A. Hewitt et al. (Dordrecht: Kluwer), 569

Whiteoak, J. B. Z. 1993, PhD Thesis, University of Sydney Whiteoak, J. B. Z., \& Green, A. J. 1996, A\&AS, 118, 329 